\title{
Integration of architectural and cytologic driven image algorithms for prostate adenocarcinoma identification
}

\author{
Jason Hipp ${ }^{\mathrm{a}, 2}$, James Monaco ${ }^{\mathrm{b}, 2}$, L. Priya Kunju ${ }^{\mathrm{a}}$, Jerome Cheng ${ }^{\mathrm{a}}$, Yukako Yagic ${ }^{\mathrm{c}}$, \\ Jaime Rodriguez-Canales ${ }^{\mathrm{d}}$, Michael R. Emmert-Buck ${ }^{\mathrm{d}}$, Stephen Hewitt ${ }^{\mathrm{d}}$, Michael D. Feldman ${ }^{\mathrm{h}}$, \\ John E. Tomaszewski ${ }^{i}$, Mehmet Toner ${ }^{\mathrm{f}}$, Ronald G. Tompkins ${ }^{\mathrm{f}}$, Thomas Flotte ${ }^{\mathrm{e}}$, David Lucas ${ }^{\mathrm{a}}$, \\ John R. Gilbertson ${ }^{\mathrm{g}}$, Anant Madabhushi ${ }^{\mathrm{b}, 1, *}$ and Ulysses Balis ${ }^{\mathrm{a}, 1, *}$ \\ ${ }^{a}$ Department of Pathology, University of Michigan, M4233A Medical Science I, Catherine, MI, USA \\ ${ }^{\mathrm{b}}$ Department of Biomedical Engineering, Rutgers The State University of New Jersey, Piscataway, NJ, USA \\ ${ }^{\mathrm{c}}$ MGH Pathology Imaging and Communication Technology (PICT) Center, Boston, MA, USA \\ ${ }^{\mathrm{d}}$ Laboratory of Pathology, National Institutes of Health, National Cancer Institute, Advanced Technology Center, \\ Gaithersburg, MD, USA \\ ${ }^{\mathrm{e}}$ Department of Laboratory Medicine and Pathology, Mayo Clinic, Rochester, MN, USA \\ ${ }^{\mathrm{f}}$ Massachusetts General Hospital, Harvard Medical School, Charlestown, MA, USA \\ ${ }^{\mathrm{g}}$ Department of Pathology, Massachusetts General Hospital, Harvard Medical School, Boston, MA, USA \\ ${ }^{\mathrm{h}}$ Department of Pathology and Laboratory Medicine, Perlman School of Medicine at the University of Pennsylvania, \\ Division of Surgical Pathology, 6 Founders Hospital of the University of Pennsylvania, Philadelphia, PA, USA \\ ${ }^{i}$ Pathology and Anatomical Sciences, School of Medicine and Biomedical Sciences, SUNY at the University of \\ Buffalo, Buffalo, NY, USA
}

Received: July 28, 2011

Accepted: February 6, 2012

\begin{abstract}
Introduction: The advent of digital slides offers new opportunities within the practice of pathology such as the use of image analysis techniques to facilitate computer aided diagnosis (CAD) solutions. Use of CAD holds promise to enable new levels of decision support and allow for additional layers of quality assurance and consistency in rendered diagnoses. However, the development and testing of prostate cancer CAD solutions requires a ground truth map of the cancer to enable the generation of receiver operator characteristic (ROC) curves. This requires a pathologist to annotate, or paint, each of the malignant glands in prostate cancer with an image editor software - a time consuming and exhaustive process.

Recently, two CAD algorithms have been described: probabilistic pairwise Markov models (PPMM) and spatially-invariant vector quantization (SIVQ). Briefly, SIVQ operates as a highly sensitive and specific pattern
\end{abstract}

\footnotetext{
${ }^{1}$ These Senior authors contributed equally.

${ }^{2}$ These First authors contributed equally.

${ }^{*}$ Corresponding author: Ulysses J. Balis, MD, Department of Pathology, University of Michigan Health System, M4233A Medical Science I, 1301 Catherine, Ann Arbor, MI 48109-0602, USA. Tel.: +1 734615 5727; Fax: +1 603250 3139; E-mail: Ulysses@med.umich.edu and Anant Madabhushi, PhD, Rutgers The State University of New Jersey, Department of Biomedical Engineering, 599 Taylor Road, Piscataway, NJ, USA. Tel.: +1 732445 4500; Fax: +1 732445 3753; E-mail: anantm@ rci.rutgers.edu.
} 
matching algorithm, making it optimal for the identification of any epithelial morphology, whereas PPMM operates as a highly sensitive detector of malignant perturbations in glandular lumenal architecture.

Methods: By recapitulating algorithmically how a pathologist reviews prostate tissue sections, we created an algorithmic cascade of PPMM and SIVQ algorithms as previously described by Doyle el al. [1] where PPMM identifies the glands with abnormal lumenal architecture, and this area is then screened by SIVQ to identify the epithelium.

Results: The performance of this algorithm cascade was assessed qualitatively (with the use of heatmaps) and quantitatively (with the use of ROC curves) and demonstrates greater performance in the identification of malignant prostatic epithelium.

Conclusion: This ability to semi-autonomously paint nearly all the malignant epithelium of prostate cancer has immediate applications to future prostate cancer CAD development as a validated ground truth generator. In addition, such an approach has potential applications as a pre-screening/quality assurance tool.

Keywords: Pathology informatics, whole slide imaging, computer aided diagnosis, SIVQ, PPMM, digital imaging, prostate cancer, cancer

\section{Introduction}

A great deal of information can be potentially extracted from H\&E-stained histologic slides. The visual interpretation of this information requires years of training by pathologists to render correct diagnoses. In the past, machine vision computational approaches (e.g. computer-aided diagnosis systems - CAD) have been developed in an attempt to interpret this information but, with the notable exception of GYN cytology screening, such efforts have usually fallen short of expectations, most notably due to lack of: algorithmic specificity, standardization of the slide making process, computational power and storage, or a properly provisioned operational setting. Additionally, prior to the advent of whole slide (WSI) technology, there were significant microscopic image field sampling limitations intrinsic to the simplistic camera/microscope paired approach of the past.

While Pap smear review represents a non-definitive screening procedure that results in a additional diagnostic procedures (such as a colposcopically-obtained cervical biopsy) upon detection of a positive event, histopathology review of tissue sections is generally considered as being a definitive diagnostic step, usually leading to significant clinical management decisions. In addition, the application of CAD to surgical pathology specimens is incrementally challenging over Pap smears in that the former requires the assessment of a number of features such as tissue architecture, anatomic frame of reference, cellular and nuclear morphology, admixed stromal changes, and/or an inflammatory response, to make a final diagnosis. Furthermore, there is often associated metadata in addition to the specimen sections, which the pathologist is similarly compelled to assess and integrate with the clinical history, patient's demographics, imaging findings, and location of the tissue sample, in the process of rendering one or more final diagnoses.

Today, with greatly improved computational capability and similarly evolved high-resolution/highthroughput WSI technologies [2-6], the image capture and analysis platforms are better, making it possible to finally consider application of CAD approaches at the whole-slide level. However, with the immense increase in histologic imagery data set size, this newfound capability to comprehensively query entire tissue sections is at the same time offset by a significant computational barrier, as resultant data set size still outpaces computational throughput. Thus, there remains significant need for highly-efficient (e.g. real time) CAD algorithms, in order to realize real-world workflow solutions, which can serve in a true decision support fashion at the time of case sign-out.

Several groups are investigating the possibility of applying CAD to prostate cancer (such as Gleason grading [7-9]) and whole slides [10]; however, this technology is currently not ready to be integrated into the work-flow of clinical practice. With increasing numbers of urologists performing saturation prostate biopsies, having CAD to pre-screen and quantify features within the slides would be of extreme benefit to practicing surgical pathologists (saturation prostate biopsies often consist of more than 20 cores, with 2-3 levels per biopsy resulting in up to $80-120 \mathrm{sec}-$ tions per patient for review). They are time-consuming and monotonous, which can potentially lead to missed diagnosis. Saturation biopsy, however, is an important clinical tool in prostate cancer for work-up of small atypical glands and low level cancers since patients with minimal tumor burden can be surveyed with this technique and spared morbid surgical operations. In 
addition, localized targeted therapy for prostate cancer is still not very widely performed due to inability of regular 12-core biopsies to detect all significant nodules of prostate cancer which is commonly a multifocal disease, could potentially be facilitated by saturation biopsies.

When a pathologist reviews a prostate cancer case (biopsies and tissue sections/whole mounts), there is a systematic logical schema of assessments (sometimes referred to as a "thought process") that is performed when evaluating the tissue sections. It is important to note that the pathologist is not a passive investigatorsuch that a pathologist reviews the tissue at low power assessing the glandular architecture and if suspicious glands are identified, actively examines them at higher power to assess the cellular morphology, in a continuous and iterative process $[11,12]$. If the high power examination is not definitive, they might choose another tool (like immunohistochemical staining, IHC) to investigate the case further. Or if the initial low power search finds nothing and if the patient has a very high, unexplained serum PSA they may choose to use another tool, such as deeper levels and/or re-cuts. The "clinical judgment" occurs when the pathologist assesses all this information to make the clinical decision of cancer or not. CAD has the potential of being another set of tools that the pathologist will use like any existing tool (like IHC and re-cuts), and like those tools, he will need to know when and how to use them and interpret their results (see Discussion below).

When designing a CAD for prostate cancer, it be would logical (and perhaps desirable) to begin by recapitulating the "low power/high power" set of assessments that pathologists currently go through when working up such a case by developing an algorithm for each of these assessments and then combining the results to get a final interpretation. The low power/high power paradigm directly maps to the need to explore successively smaller length scales, towards obtaining a unified computational interpretation. An algorithm that seeks to mimic the "low to high power" analysis used by pathologist would have to 1) be able to handle pattern recognition at multiple spatial scales and 2) be fast enough to cover the large areas implied by a low power analysis.

One such recently reported algorithm, which can identify specific morphologies across multiple length scales, is termed Spatially-Invariant Vector Quantization [13-15]. Briefly, SIVQ is a pattern matching algorithm that can be used to match patterns across a range of length scales, including nuclear, cellular and architectural features (e.g., capable of matching at high, medium and low magnifications, respectively) [13]. SIVQ, as a pattern matching algorithm, differs from other image analysis approaches in that it allows for significantly greater matching speed. This feature alone, in turn, allows for an interactive discovery workflow model. Additionally it's utility as a discovery tool is further amplified by its simplified user interface and uncomplicated user training requirements. Taken together, these attributes embody a turn-key platform for general feature selection and pattern recognition tasks, as encountered by non-technical biomedical subject matter experts (SMEs). For example, a predicate image feature could be identified by a user, with this predicate then available to search for possible matches within the remaining image (or a library of images, for that matter), resulting in the generation of a statistical probability heat map of equivalency Figs. 2A, 3A, 4A).

While unambiguously a pattern matching algorithm, SIVQ fundamentally differs from the vast plurality of extant approaches in that its search operators, with no particular angular or positional orientation, leverage the continuous symmetry of a circle - the only construct in nature that possesses such symmetry. As already reported [13], the ring vectors of SIVQ overcome the stochastic sampling limitations of conventional grid-based vectors including: translationally, rotationally, and chirally. The net effect of this spatial invariance is that a single ring vector of SIVQ can replace the need for millions of possible stochastically derived Cartesian vectors, still yielding equivalent performance. Thus, a concentric ring vector set can collapse the candidate vector pool to often as few as a single cohort of rotationally-coupled ring vectors. While SIVQ can operate at multiple length scales (high and low power), identifying vectors to capture features such as lack of a basal cell layer (which is a hallmark of prostate cancer) and/or various luminal shapes is quite challenging. For example, designing a vector to identify basal cells can be difficult as basal cells are often distributed in a patchy and/or discontinuous manner in benign mimics of cancer. In day to day surgical practice, basal cell immunohistochemical markers such as $34 \beta E 12$ and/or p63 are frequently used in the work-up of atypical glands to confirm the presence or absence of basal cells. Therefore, we sought to integrate SIVQ with an algorithm whose strength is in identifying architectural perturbations of prostate cancer. 
A recently described image analysis algorithm that assesses luminal architecture using Markov random fields was introduced in Monaco et al. [12]. Markov models provide a Bayesian mechanism for incorporating contextual information. For example, Markov models can inherently increase the probability that a region (e.g., pixel) is cancerous when regions (e.g., pixels) that neighbor it have a high probability of being cancerous. Note that SIVQ does not consider such contextual information when performing classification. However, integrating Markov models into SIVQ is quite possible.

The Markov system introduced by Monaco et al. [12] was the first system for rapidly detecting carcinoma regions in whole-mount H\&E stained histological sections from radical prostatectomies. Their detection system requires less than two minutes to process an entire whole-mount image on a standard PC desktop computer. They achieved this in a high throughput manner by tailoring the algorithm to accurately analyze the histological section at low resolution. For even at low resolution, gland size and morphology remain noticeably different in cancerous and benign regions [12]. This motivated the following biologically driven algorithm: Step 1) glands are identified and segmented, Step 2) the segmented glands are classified as malignant or benign, and Step 3) the malignant glands are consolidated into continuous regions indicating the spatial extent of cancer. The classification of individual glands (Step 2) leverages two simple, but effective, features of biological relevance: 1) glands size and 2) the tendency for proximate glands to share the same class. The second feature describes a spatial dependency that exists among the glands. This dependency is modeled using probabilistic pairwise Markov model (PPMM) ${ }^{1}$, a novel type of Markov random field.

The evaluation of CAD solutions requires a gold standard to compare, contrast and improve diagnostic performance. This requires a pathologist to annotate an image to identify cancer and non-cancerous areas. Traditionally, this is done where a pathologist circles the tumor nodule on the digital slide. However, in the case of prostate cancer, when tumor nodules are circled, it often includes benign stroma and lumenal white space. Thus, if performing a pixel-by-pixel analysis,

\footnotetext{
${ }^{1}$ Note the PPMM will be used to refer to the entire algorithm for detecting carcinoma in histological sections, and not only to the Markov models that this algorithm employs.
}

such as by SIVQ, these benign areas would be annotated as cancer. Therefore, the ideal annotation would be done on a cell by cell basis, but human annotation at this level is usually prohibitively time and resource intensive. Thus, having a CAD algorithm that could aid in the "painting" of the majority of the tumor epithelium would enable the pathologist to spend more time on the more challenging and critical task of identify the atypical/suspicious glands and on Gleason grading [16-19].

By recapitulating algorithmically how a pathologist reviews prostate tissue sections using an algorithmic cascade of PPMM and SIVQ, where PPMM identified the glands with abnormal lumenal architecture, and this area was then screened by SIVQ to identify the epithelium. We qualitatively and quantitatively show that this approach improves the identification of malignant prostate glands.

\section{Materials and methods}

\subsection{Images}

Three WSI data sets from the Monaco et al. [12] data set were analyzed for this study; for a detailed description the reader is directed to Monaco et al. [12] (IRB \#E09-481). Briefly, whole mount histological sections of the prostate gland were cut into 4 quadrants for three prostatectomy specimens which were then formalin fixed and paraffin embedded. H\&E stained tissue section were subsequently digitized at $20 \times$ magnification ( 0.25 um per pixel) via an Aperio ScanScope XT scanner, as previously described. A digital slide from three separate prostatectomy specimens, representing one quadrant with prostate cancer, was used in this study. Pathologists at the University of Pennsylvania circled the cancerous regions using Aperio's ImageScope ${ }^{\mathrm{TM}}$ software tool. Ground truth maps of annotated malignant epithelium were painted by pathologists at the University of Michigan using the GNU Image Manipulation Program (GIMP, www.gimp.org).

For the PPMM and SIVQ analysis, the SVS files were down-sampled $1 / 16$ and $1 / 4$ respectively to decrease the time for computational analysis.

All images reported here are available at the WSI repository (www.WSIrepository.org) as described by Hipp et al. [20, 21]. 


\subsection{Spatially-Invariant Vector Quantization (SIVQ)}

SIVQ is unique in that it uses a set of rings instead of a block. A ring is the only geometric structure in two-dimensional space besides a point that exhibits continuous symmetry. With the use of a series of concentric rings, it is possible to reduce the total set of potential matches, intrinsic to a two dimensional orientation problem, into a greatly simplified linear pattern matching problem, where individual rings are iteratively assessed, along all possible rotational configurations. Greater specificity in feature matching can be addressed by creating a family of concentrically-nested sub-rings, which all rotate in tandem, as iteratively searching is performed. Sensitivity of this construct can be enhanced by allowing for relaxation of the rotational lock, thus allowing for rotational "wobble" between adjacent rings, which in turn, allows for greater likelihood of pattern patching between predicate and candidate features. With increasing wobble comes increasing sensitivity. Lastly, adjusting the ring diameter allows for selection of candidate feature from different length scales; constellations of such variegated ring vectors allows for the creation of compound sets of vectors that exhibit enhanced specificity and sensitivity, potentially across a number of image feature classes and length scales (allowing for concurrent gating of both tissue architecture and cytologic features). For further details, please refer to Hipp and Cheng et al. [13].

\subsection{Probabilistic Pairwise Markov Model (PPMM)}

This model was previously described in Monaco et al. [12] with the following modification. First, the glands were segmented and then labeled as malignant or benign based on their area and their proximity to other malignant/benign glands. The sensitivity of the system can be adjusted by modifying the value of the user-defined parameter $\alpha \in[0,1]$. To associate a probability of malignancy with each gland we first determine the results of the CAD system for all $\alpha \in$ $\{0,1 / 200,2 / 201, \ldots, 1\}[12]$. For each gland, we then record the fraction of these 201 values for which the CAD system labels it (i.e., the gland) as malignant; this fraction is the probability of malignancy. To create a pixel-wise probability measure, each pixel was assigned a weighted average of the probabilities of all the surrounding glands. Specifically, the contribution (weight) of each gland was generated from a Gaussian function of distance from the centroid of that gland to the pixel under consideration.

The output of the PPMM analysis was painted onto the image. The statistical threshold selected for matching events was empirically adjusted to optimize for sensitivity.

\subsection{PPMM prescreening followed by SIVQ}

The PPMM algorithm was run to identify suspicious areas with abnormal lumenal architecture and was adjusted to optimize for sensitivity. All of the areas that were selected by PPMM were then fed to SIVQ. A single vector from each case (total of 3 vectors for the 3 cases, vector size ranging from 4-7 pixels in diameter and selected to capture the hyperchromatic nuclei and adjacent glandular cytoplasm) was used to analyze the PPMM selected areas and identify the malignant epithelium.

\section{Results}

\subsection{Histopathologic analysis as determined by a pathologist}

High resolution digital images from whole slide scanning of H\&E-stained whole-mount prostate tissue sections from the data set of Monaco et al. [12] were used for this study. The areas of cancer had been previously annotated (circled in red) by pathologists as shown in Fig. 1A, C, E. The images were also reviewed by pathologists at the University of Michigan and the malignant epithelium was annotated (painted in green) as shown in Fig. 1B, D, E).

The first case shows an area of prostatic adenocarcinoma (Gleason Grade $3+3=6$ ) in three foci in the lower left corner (Fig. 1A). The second case has a tumor nodule in the lower right hand corner that is a Gleason Grade $4+3=7$ (Fig. 1C). The third case contains 4 tumor foci in the middle left and bottom center consisting of a Gleason Grade $3+3=6$ pattern Fig. 1E). Note that since PPMM leverages luminal architecture to detect cancerous regions, the algorithm can have difficulty detecting high-grade cancer (e.g., grade 5), which may not produce lumina. This limitation was a deciding factor in our choice of test images. 


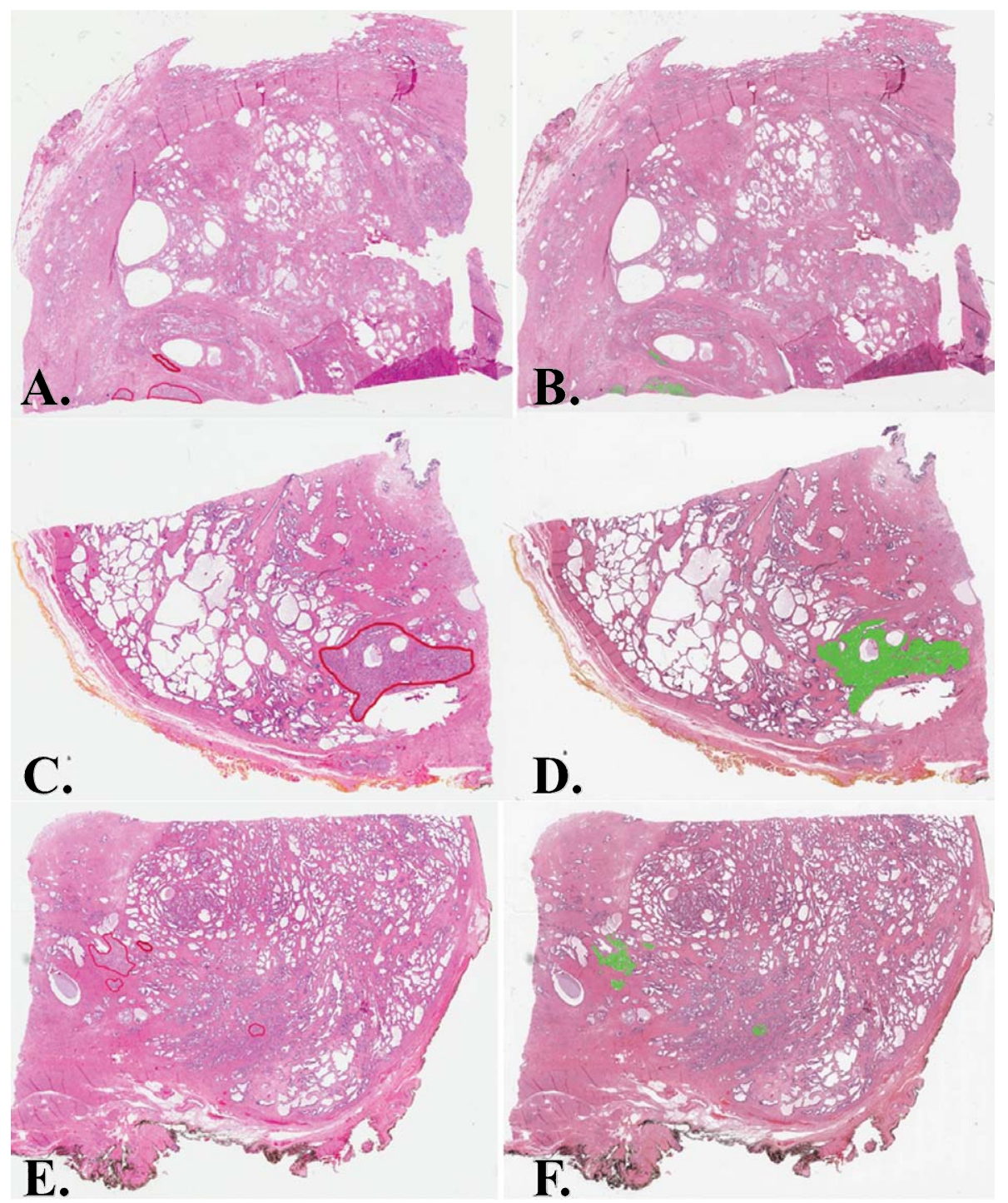

Fig. 1. Ground truth maps of the H\&E stained prostate tissue section. Digital slides from the Monaco et al. [12] data set were used for this study. $3 \mathrm{H} \& \mathrm{E}$ stained prostatic tissue sections had been scanned into digital slides. The cancerous areas were annotated (circled) by pathologists in red (A, C, E). Additionally, the malignant prostatic epithelium was annotated (painted) by a pathologist in green (B, D, F). (Colours are visible in the online version of the article; http://dx.doi.org/10.3233/ACP-2012-0054)

\subsection{Spatially-Invariant Vector Quantization analysis: To identify prostatic epithelium}

Ring vectors, containing a portion of the hyperchromatic nuclei and adjacent glandular cytoplasm (see insets of Figs. 2A, 3A, 4A) were selected from the malignant epithelium and used to analyze the entire tissue section for each case. The resultant threshold map is shown in Figs. 2B, 3B, 4B. This resulted in the identification of large areas of both benign and malignant epithelium in all 3 samples.

\subsection{Probabilistic Pairwise Markov Model analysis: To identify suspicious regions based on abnormal lumenal architecture}

PPMM - which, as mentioned previously, refers to the entire $\mathrm{CaP}$ detection process, and not simply the Markov model - was performed as described by Monaco et al. [12]. For the first sample, PPMM identified two of the three cancer foci (Fig. 2B). It also identified the focus in the upper left of crowded, lobulated small benign atrophic glands (supplemen- 

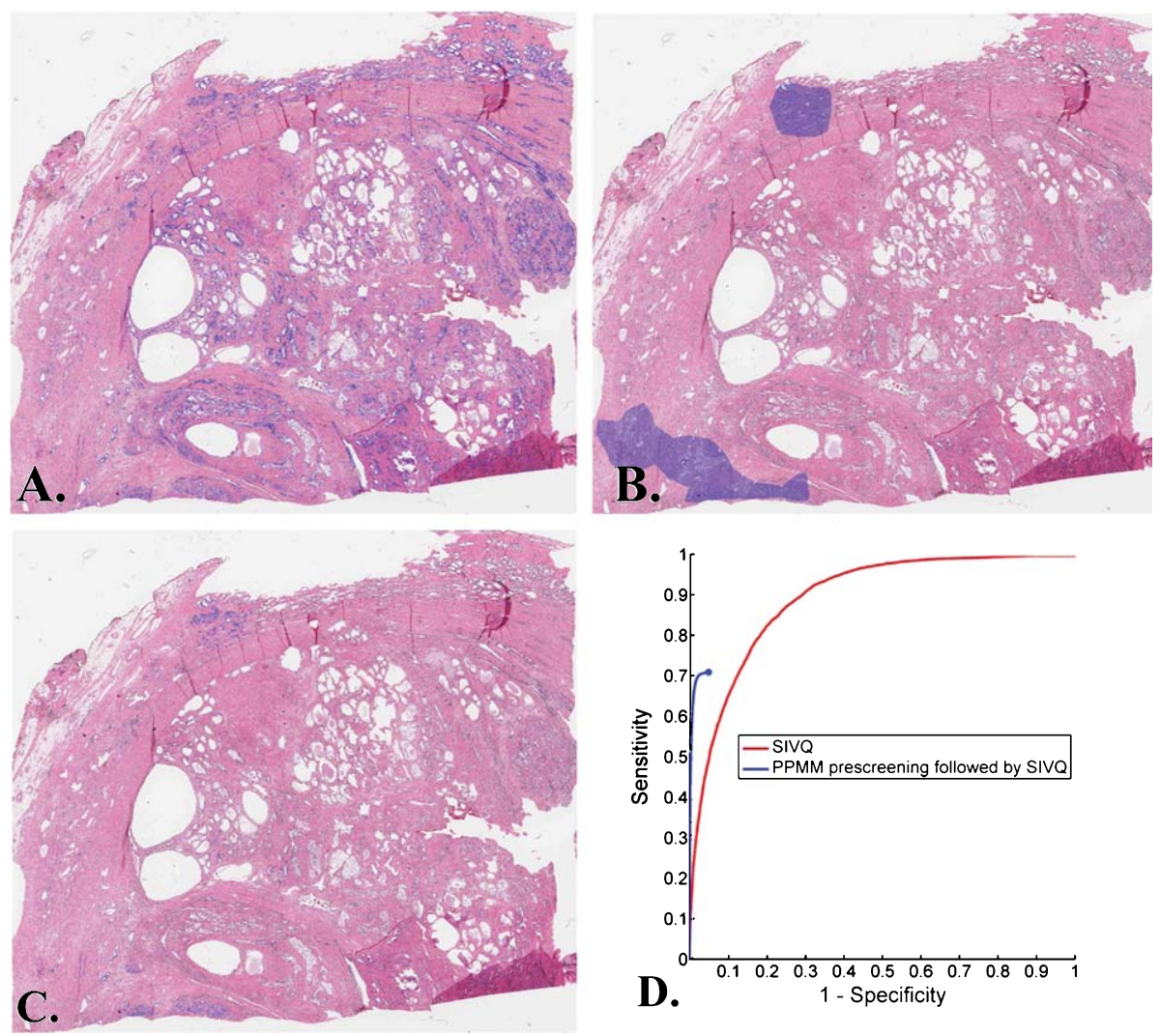

Fig. 2. SIVQ, PPMM, PPMM-SIVQ analysis of sample \#1. Using SIVQ, a ring vector was selected to identify the prostatic epithelium. The resultant thresholded heatmap is shown in Panel A. Nearly all the epithelium was identified. The same specimen was then analyzed with PPMM. The resultant thresholded heatmap is shown in Panel B. PPMM identified 2 out of the 3 cancer foci. The algorithm cascade of PPMM followed by SIVQ was then used to analyze the specimens. The malignant epithelium within 2 of the 3 cancer were identified and annotated in Panel C. To quantitatively assess and compare the performance of SIVQ to PPMM-SIVQ, an ROC curve was generated and shown in Panel D (SIVQ is red, PPMM-SIVQ is blue). PPMM-SIVQ far exceeds the performance of SIVQ alone when compared over the range of specificities achieved by PPMM-SIVQ Note that since PPMM-SIVQ applies SVIQ only to a subset of the tissue (which is determined by PPMM), its performance is bounded by the maximum sensitivity and minimal specificity established by this subset. This explains why the PPMM-SIVQ ROC curve terminates abruptly. (Colours are visible in the online version of the article; http://dx.doi.org/10.3233/ACP-2012-0054)

tal Figure 1A) and a few atrophic glands just above and to the left of the two tumor foci at the bottom (Table 1).

In the second sample, PPMM identified nearly the entire tumor nodule in the lower right; however, it did not identify the malignant glands that were above the severely dilated benign glands (Fig. 3B). It also identified at the very bottom of the tumor nodule, a focus of medium and large acini that looked very similar to the tumor nodule. Upon further review, it is favored that these glands are malignant and would need immunohistochemistry stains to confirm (supplemental Figure 1B). In addition, it identified adjacent prostatic intraepithelial neoplasia (PIN) at the far left of the tumor nodule (supplemental Figure 1C) (Table 1).
In the third sample, PPMM identified three of the 4 tumor foci (Fig. 4B). It identified two additional foci in the lower right hand corner of the specimen that had artificial white tissue spaces in the stroma (supplemental Figure 1D). While it identified the tumor foci in the middle of the specimen, it also identified numerous crowded, lobular, large and small benign glands (Table 1).

\subsection{PPMM prescreening followed by SIVQ (PPMM-SIVQ)}

Using an algorithmic cascade approach [1], the areas identified by the PPMM algorithms suspicious area 
Table 1

Qualitative summary of the results from the PPMM vs. PPMM-SIVQ analysis

\begin{tabular}{|c|c|c|c|}
\hline $\begin{array}{l}\text { A } \\
\text { Sample \# }\end{array}$ & Pathologists diagnosis & PPMM: True positive & PPMM: False positive \\
\hline 1 & 3 foci of cancer & Found 2 of 3 foci of cancer & $\begin{array}{l}1 \text { area of crowded benign atrophic glands } \\
\text { falsely called cancer } \\
\text { Few atrophic acini adjacent to cancer }\end{array}$ \\
\hline 2 & 1 nodule of cancer & $\begin{array}{l}\text { Found almost the entire nodule of } \\
\text { cancer }(>90 \%)\end{array}$ & $\begin{array}{l}1 \text { area suspicious for cancer } \\
1 \text { area of PIN }\end{array}$ \\
\hline 3 & 4 foci of cancer & Found 3 of 4 foci of cancer & $\begin{array}{l}2 \text { foci of artifactual space in stroma } \\
\text { Identified areas of crowded lobular } \\
\text { benign glands close to cancer }\end{array}$ \\
\hline \multicolumn{4}{|l|}{ B } \\
\hline Sample \# & Pathologists diagnosis & PPMM-SIVQ: True positive & PPMM-SIVQ: False positive \\
\hline 1 & 3 areas of cancer & $\begin{array}{l}\text { Identified the malignant } \\
\text { epithelium in } 2 \text { of the } 3 \text { areas of } \\
\text { cancer identified by PPMM }\end{array}$ & $\begin{array}{l}\text { Identified the epithelium of } 1 \text { area of } \\
\text { crowded benign atrophic glands } \\
\text { Identified the epithelium of few atrophic } \\
\text { acini adjacto to tumor nodule }\end{array}$ \\
\hline 2 & 1 area of cancer & $\begin{array}{l}\text { Found almost entire nodule of } \\
\text { cancer }>90 \%\end{array}$ & $\begin{array}{l}1 \text { area suspicious for cancer } \\
1 \text { area of PIN }\end{array}$ \\
\hline 3 & 4 areas of cancer & Found 3 of 4 foci of cancer & $\begin{array}{l}\text { Identified areas of crowded lobular } \\
\text { benign glands close to cancer }\end{array}$ \\
\hline
\end{tabular}

The far column of Panel A corresponds to the sample number. To the right of this is a qualitative description of the pathologists diagnosis. To the right of this is a qualitative description of the PPMM true positive results. The final column on the right is a qualitative description of the PPMM false positive results; The far column of Panel B corresponds to the sample number. To the right of this is a qualitative description of the pathologists diagnosis. To the right of this is a qualitative description of the PPMM-SIVQ true positive results. The final column on the right is a qualitative description of the PPMM-SIVQ false positive results.

for cancer were selected and subsequently analyzed by SIVQ. For sample 1, PPMM-SIVQ identified the malignant epithelium within the two of the three cancer foci that was identified by PPMM (Fig. 2C). It also identified the focus in the upper left of crowded, lobulated small benign atrophic glands (supplemental Figure 1A) and a few atrophic glands just above and to the left of the two tumor foci at the bottom (Table 1).

In the second sample, PPMM-SIVQ identified the malignant epithelium within the tumor nodule identified by PPMM. It also identified the suspicious focus of acini at the very bottom of the tumor nodule (supplemental Figure 1B) and the PIN adjacent to the far left of the tumor nodule (supplemental Figure 1C) as described above in the PPMM section (Table 1).

In the third sample, PPMM-SIVQ identified the malignant epithelium within three of the 4 tumor foci. However, it did not identify the two foci of artificial white tissue spaces in the stroma at the lower right hand corner of the specimen (supplemental Figure 1D). While it identified the tumor foci in the middle of the specimen, it also identified crowded, lobular, large and small benign glands. In addition, upon further review, another minute focus of small and large highly suspicious glands (which was not mapped) was not identified by PPMM/PPMM-SIVQ (supplemental Figure 1E) (Table 1).

\subsection{Quantitative assessment of SIVQ and PPMM-SIVQ performance}

The output of the SIVQ analysis is a value indicative of a quality of match, from 0-255, enabling the generation of ROC curves for the SIVQ and PPMMSIVQ analysis (Figs. 2D, 3D, and 4D). PPMM results in a binary output (either the pixel value is cancer or no cancer) and a single point in the graph indicates its sensitivity and specificity. For the PPMM-SIVQ analysis, because SIVQ is performed only on the regions identified by PPMM, it can only identify those cancer glands that PPMM identified. This explains why the PPMMSIVQ ROC curves terminates early: PPMM-SIVQ is bounded by the maximum sensitivity and minimum specificity as established by preliminary PPMM step.

For all the samples, the PPMM-SIVQ far exceeded the SIVQ alone ROC curve. The PPMM-SIVQ analysis had approximately $90 \%$ sensitivity, $90 \%$ specificity 

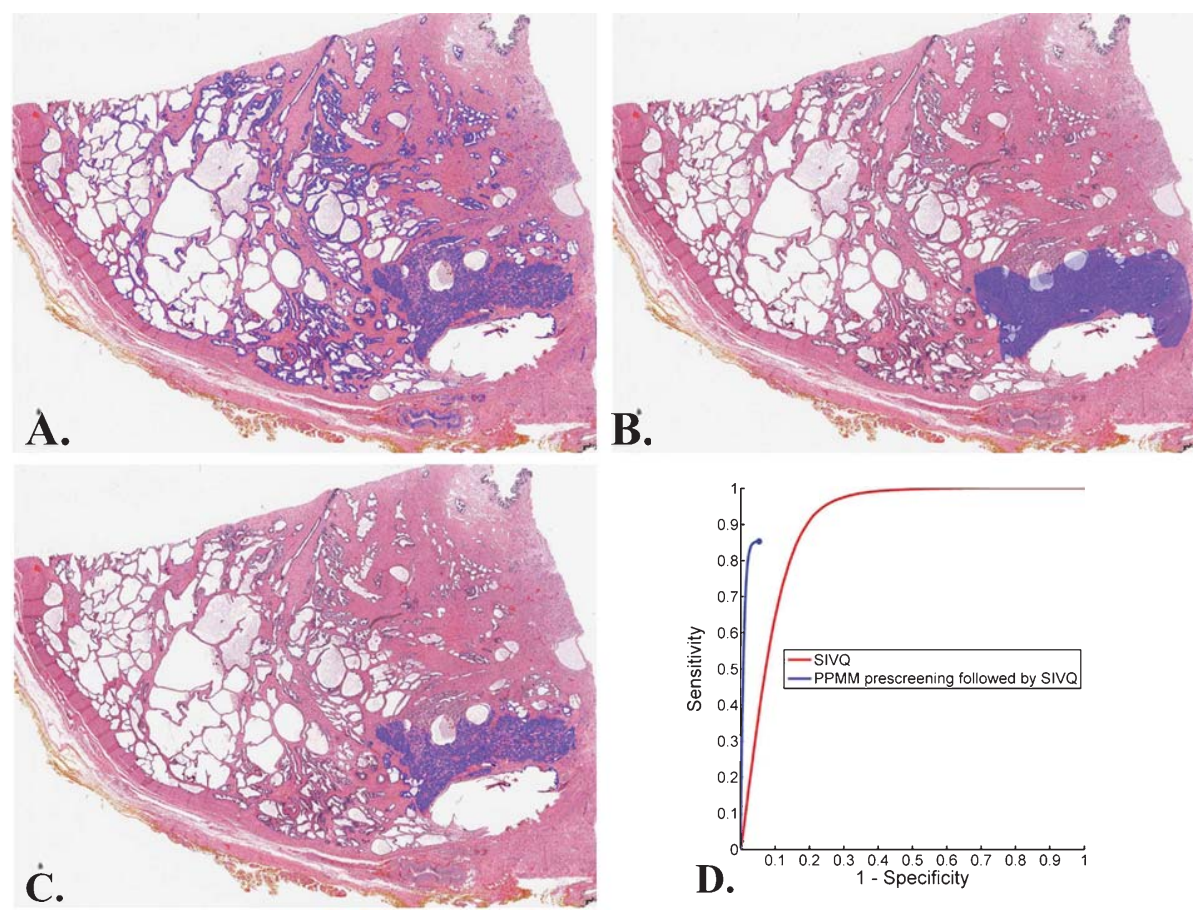

Fig. 3. SIVQ, PPMM, PPMM-SIVQ analysis of sample \#2. Using SIVQ, a ring vector was selected to identify the prostatic epithelium. The resultant thresholded heatmap is shown in Panel A. Nearly all the epithelium was identified. The same specimen was then analyzed with PPMM. The resultant thresholded heatmap is shown in Panel B. PPMM identified nearly the entire tumor nodule. The algorithm cascade of PPMM followed by SIVQ was then used to analyze the specimens. The malignant epithelium within the majority of the tumor nodule was identified and annotated in Panel C. To quantitatively assess and compare the performance of SIVQ to PPMM-SIVQ, an ROC curve was generated and shown in Panel D (SIVQ is red, PPMM-SIVQ is blue). PPMM-SIVQ far exceeds the performance of SIVQ alone when compared over the range of specificities achieved by PPMM-SIVQ. (Colours are visible in the online version of the article; http://dx.doi.org/10.3233/ACP-2012-0054)

(Sample \#1), 85\% sensitivity, 94\% specificity (Sample \#2), and $90 \%$ sensitivity, $88 \%$ specificity (Sample \#3).

Since the ROC curves of PPMM-SIVQ end at false positive rate (i.e., 1 - specificity) below 1 , the areas under the ROC (AUCs) were determined over the valid range and then normalized by the maximum achievable value. The normalized AUCs for SIVQ/PPMM-SIVQ for the three samples are: Sample \#1 (0.3330/0.6586), Sample \#2 (0.1872/0.7134), and Sample \#3 (0.1808/0.6451).

To assess the consistency and reproducibility of using vectors derived from separate specimens, each of the specimens were then analyzed with the three ring vectors from above. In Fig. 5, the red curves represent the ROC curves from just the SIVQ analysis. Since these vectors were designed to identify epithelium, and were of different ring sizes (4-7 pixels in diameter), their performance variability was expected to identify benign and malignant epithelium. PPMM followed by SIVQ was used to analyze the three cases for each of the three vectors and their ROC curves (in blue) are shown in Fig. 5. The PPMM-SIVQ curves show a consistent and reproducible increase in performance independent of which vector was used compared to SIVQ alone.

\section{Discussion}

In this study, we have shown that a cascade approach as described by Doyle et al. of PPMM followed by SIVQ algorithms essentially recapitulates algorithmically how a pathologist reviews prostate tissue sections. In essence, PPMM provides the CADequivalence of low power assessment (recapitulating glandular architecture assessment by pathologist), with SIVQ providing cytologic assessment (similar to assessment of the pathologist at "high- power"). Together, this approach represents a CAD-domain multi-length-scale solution which appears to be effective at recapitulating the cognitive processes invoked by experienced diagnosticians. 

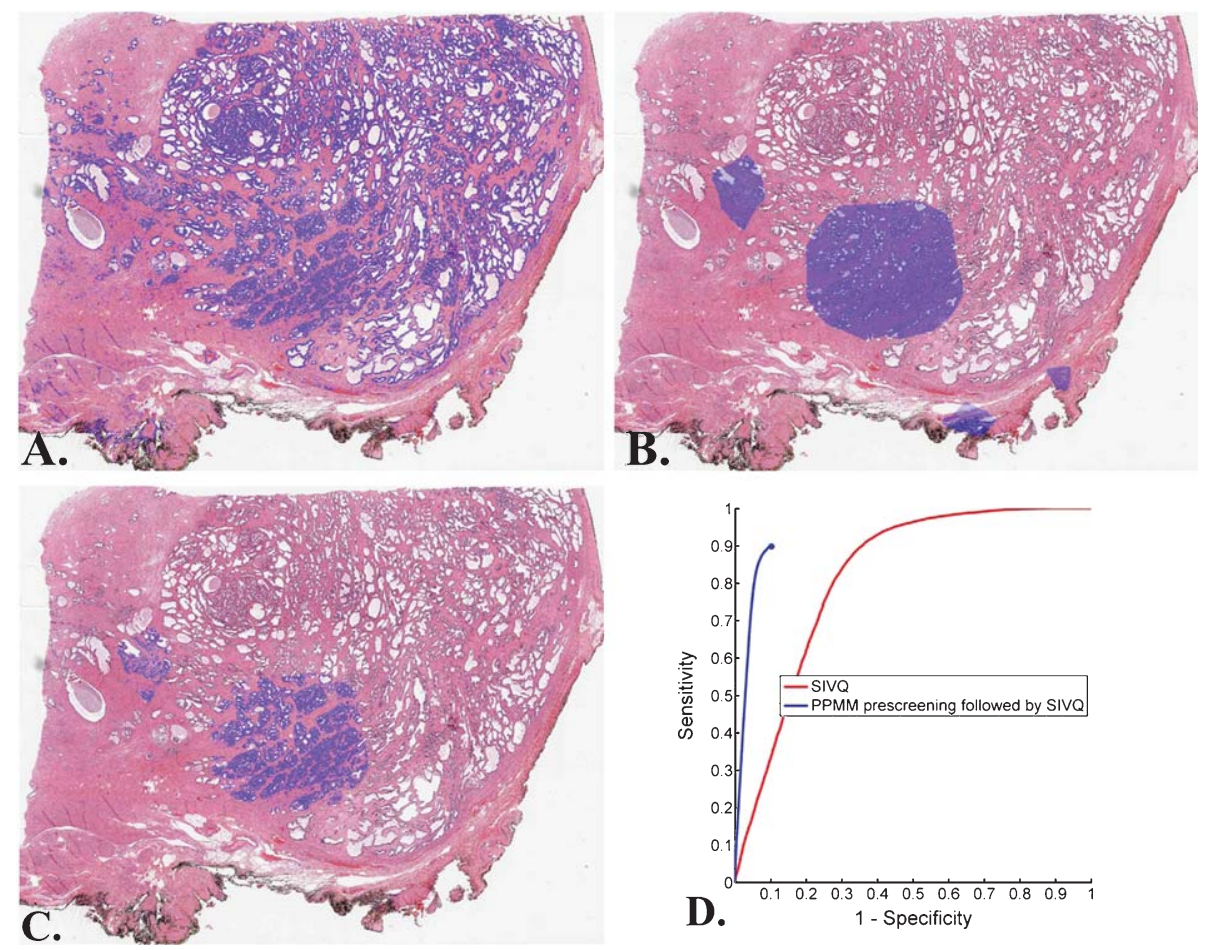

Fig. 4. SIVQ, PPMM, PPMM-SIVQ analysis of sample \#3. Using SIVQ, a ring vector was selected to identify the prostatic epithelium. The resultant thresholded heatmap is shown in Panel A. Nearly all the epithelium was identified. The same specimen was then analyzed with PPMM. The resultant thresholded heatmap (binary mask) is shown in Panel B. PPMM identified 3 of 4 tumor foci. The algorithm cascade of PPMM followed by SIVQ was then used to analyze the specimens. The malignant epithelium within the majority of 3 of the 4 tumor foci was identified and annotated in Panel C. To quantitatively assess and compare the performance of SIVQ to PPMM-SIVQ, an ROC curve was generated and shown in Panel D (SIVQ is red, PPMM-SIVQ is blue). PPMM-SIVQ far exceeds the performance of SIVQ alone. (Colours are visible in the online version of the article; http://dx.doi.org/10.3233/ACP-2012-0054)

This synthesis leverages the strengths of each algorithm, both architectural and cytological, such that each algorithm independently assesses fundamentally different properties of prostate cancer (with SIVQ identifying the epithelial morphology and PPMM identifying abnormal lumenal architecture). This semi-automated approach yields improved identification of specifically the malignant epithelium, with the immediate consequence being a plurality of immediately realizable applications to further prostate cancer CAD development, specifically by improving the efficiency of ground truth mapping. In addition, such an approach has potential application as a pre-screening/quality assurance tool.

In general, PPMM alone was very successful in identifying the majority of the tumor foci. Since PPMM analyzes only the white spaces and operates based on the size and shape of the white space and its proximity to malignant glands, not surprisingly, PPMM would identify clusters of small atrophic glands and artifactual tissue spaces in the stroma, which from the perspective of PPMM would appear to be indicative of cancerous glands (abnormal white space within the lumens). For sample \#2, the source of PPMM not identifying the top of the tumor nodule was due to the 2 large, severely dilated benign glands creating a sufficiently large space as to preclude the Markov detection filter from recognizing malignant glands beyond those represented by large benign acini.

The PPMM approach classifies cancerous regions simply as a function of their adjacency, with this classification being fully independent of any intrinsic property to local texture or luminance - with these areas being, in essence, "guilty by proximal association". This classification behavior is exactly what would be expected from a Markovian Model, which builds towards classification certitude based on a "preponderance of local domain evidence". Finally, it should be noted that an additional reason for PPMM's resistance to excluding the altered morphology of 

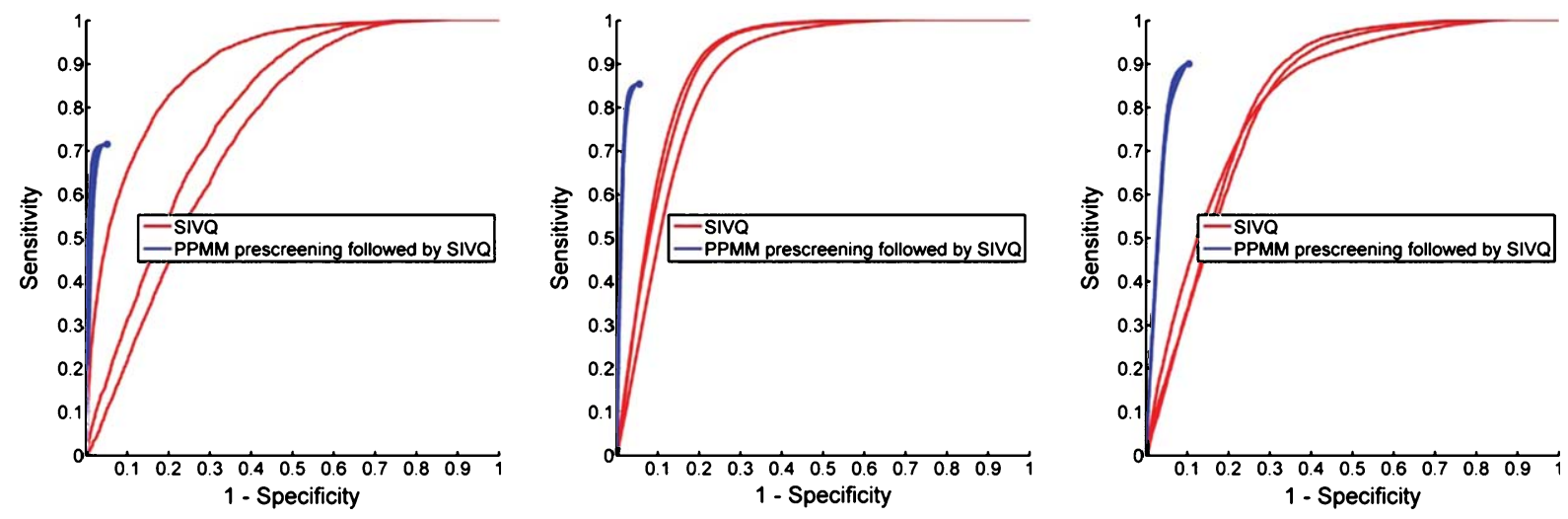

Fig. 5. Quantitative analysis and comparison of using vectors derived from other specimens. To assess the consistency and reproducibility of using vectors derived from separate specimens, each of the specimens were then analyzed with the three ring vectors from above. The red curves represent the ROC curves from just the SIVQ analysis. Since these vectors were designed to identify epithelium, and were of different ring sizes (4-7 pixels in diameter), their performance variability was expected to identify benign and malignant epithelium. PPMMSIVQ was used to analyze the three cases for each of the three vectors and their ROC curves (in blue) are shown above (left correspond to specimen \#1, middle specimen \#2, and right specimen \#3). The PPMM-SIVQ curves show a consistent and reproducible increase in performance independent of which vector was used compared to SIVQ alone. As mentioned previously, since PPMM-SIVQ applies SVIQ only to a subset of the tissue (which is determined by PPMM), its performance is bounded by the maximum sensitivity and minimal specificity established by this subset. This explains why the PPMM-SIVQ ROC curves terminate abruptly. (Colours are visible in the online version of the article; http://dx.doi.org/10.3233/ACP-2012-0054)

tissue processing artifacts, such as folds, is its dependence on essentially only the pattern of white lumenal spaces of glands, and not tissue/textural information.

SIVQ is a pattern recognition algorithm whose strength lies in identifying textural features (in this case, specifically at the cytologic length scale). However, prostate cancer is not a cytologic diagnosis alone, but rather, includes a constellation of both cytologic (nuclear atypia including prominent nucleoli) and architectural features (absence of a basal layer, crowded glands with abnormal, infiltrative lumenal architecture, glandular retraction, intralumenal contents). Therefore, attempts to create a vector capable of recognizing nucleoli specific to malignant glands failed, owing to the observation that not all malignant glands exhibit identical prominent nucleoli. Secondly, it required the analysis to be performed at 20x magnification, with such analysis at this length-scale requiring significantly greater computational time (on the order of days on even a high-performance dual or quad core workstation) for as few as a single digital slide.

When testing vectors derived from one specimen on other specimens, we show variable performance in the resultant ROC curves. This was expected because the ring vectors were generic in order to identify the epithelium, and that the epithelial morphology differs across cases and between benign and malignant features. However, by prescreening with PPMM, this identified the malignant areas which thus constrained down the variability of the epithelia morphology, as demonstrated by the blue curves in Fig. 5 .

In this study, we used a cascade approach where the PPMM was used to truncate the suspicious areas and SIVQ was analyzed only on this "cut out" digital region of interest. We found this approach best recapitulated the process by which pathologist reviews prostatic tissue, with it combining the discriminant strengths of both the PPMM and SIVQ algorithms. Screening is initially performed at low power (to assess architectural features) and suspicious areas are subsequently examined at higher power to assess for cytologic features. While this study highlights the advantages of using an integrated algorithmic approach (lumenal architecture and cytology), we envision adding additional algorithms and intervening length scales to assess other unique architecture and nuclear features of the prostate cancer described above. Moreover, this cascaded approach can be easily tuned to similarly recapitulate the exact diagnostic process carried out by a surgical pathologist, for each posed diagnostic challenge.

Additionally the findings confirm that added diagnostic power is made possible by algorithm integration. Along this line of questioning, we explored other combinational methods such as use of PPMM and SIVQ approaches independently, and then combining their 
initial results by a local kernel point-wise convolution. Given that the SIVQ and PPMM techniques matched areas within tissue and intra-lumenal open spaces, respectively, their inherent direct overlap was minimal, with this reality diminishing the merit of a point-wise cross-multiplication convolution operator. Rather, we addressed the orthogonality of algorithm selectivity by applying a ninth-order Gaussian point spread function to both images, allowing for the creation of overlapping regions where the statistical power of each individual algorithm could be boosted in synergy. This spatial juxtaposition of the two matching regions is mathematically viable if we assume that they are independent events. Thus, the Gaussian operator serves essentially in the role of a specialized Markov field discriminant.

\subsection{CAD on prostate whole mounts}

One can envision using this technology on prostate whole mounts in a prescreening role to aid the pathologist in identifying cancerous regions. While it would be tempting to perform such analysis on saturation prostate biopsy cases, doing so poses a much greater challenge because of the relatively few number of glands, with this diminution degrading the performance of the overall Markov modeling process (which is dependent upon the adjacency of a critical mass of malignant glands). One potential solution would be to use the "blurring" integration approach already described above, which would give more emphasis to the cytologic and histologic features, rather than adjacency of glands. Finally, in settings where there is less total tissue, it would be practical to run SIVQ at a higher magnification.

Recent studies have shown prostate tumor volume to be an independent predictor of recurrence and longterm survival in multivariate analysis studies $[25,26]$. Unfortunately, the inability to arrive at a standard means for measuring tumor volume [24, 25, 27] has resulted in articles both extolling and challenging its prognostic utility [28]. Another potential application of the PPMM-SIVQ technique would be in measuring tumor volume. Such an approach would be more precise than the current methodology, which extrapolates upon a linear maximal tumor dimension. We have previously shown accurate and precise surface area measurement with SIVQ [14]. One can extrapolate this approach to measure the surface area of paint from the PPMM-SIVQ analysis. These 2-dimensional painted whole mount sections could conceivably be reconstructed into 3 dimensional images. However, registration can be a difficult task, complicated by factors such as rotated or shifted slides, tissue deformation, tissue folding, tissue loss during the sectioning, and variable spacing between sections. Please see Xiao et al. [29] for a list of the major hurdles.

\subsection{The importance of a composite approach}

There is value in recognizing the intrinsic limitations of such tools when used alone and similarly, how their use in composite constructs allows for computer-aided enhancement of both detection and classification of important regions of interest.

When comparing the classification performances of these two disparate approaches, SIVQ emerges as the algorithm with the smaller feature detection length scale (equivalent to a high power view), while PPMM operates at a larger length scale (low power view). Each approach alone has the potential for false-positive and false-negative gating, based upon the limitations of cancer detection at each length scale. However, when implemented as a composite detection construct, the two approaches complements each other, with the notable result being that use of appropriately-nested Boolean "OR" and "AND" operations of the two result classes can generate a derived result that exhibits significantly better accuracy than use of either algorithm alone.

When considering that the PPMM approach is possibly the current gold standard for classification of cancerous areas in whole-slide imagery, the above composite construct shows improved performance in identification of malignant prostatic epithelium, and may serve as a validated ground truth generator for malignant prostate epithelium.

\subsection{Potential integration into the clinical workflow}

Another significant factor in the clinical utility of $\mathrm{CAD}$ algorithms is the ease with which they can be incorporated into pathology information systems and the associated laboratory workflow. Most every contemporary anatomic pathology laboratory information system (AP-LIS) supports "Part Types" or 
Specimen Types" with these allowing laboratories to apply the principle of "standard work" to similar types of specimens. As an example, laboratories routinely define a Prostate Biopsy Part Type and use this definition to constrain the standard workup for prostate biopsies. This workup might include: the submission protocol for each biopsy into one or more cassettes, the specific gross dictation protocol, and finally, the specific tissue processing, cutting and staining protocols (i.e., five slides, stepped, with the first, third and fifth slide stained with $\mathrm{H} \& \mathrm{E}$ and the remainder stored unstained). Increasingly, modern AP-LIS solutions allow labs to extend these basic, part-type-driven protocols to include imaging and image analysis metadata. In the current example, the "Prostate Biopsy" Part Type workup would include an imaging protocol and one or more image analyses diagnostic segments (on the three stained slides) with the results available through the LIS schema. Slides would then be sent to the pathologist with a note in the pathologist work queue (or printed on the case paper work) indicating that images and associated image analysis data are also available). As WSI devices become increasingly reliable and faster (leading to the ability to render a WSI dataset from a single prostate biopsy slide in less than three minutes) this approach will become practicable. The duration of running the image analysis algorithms alone is as follows: PPMM rendering in less than 2 minutes per image and the SIVQ analysis on the PPMM identified regions took about 15 minutes.

\subsection{Ground truth for prostatic adenocarcinoma}

The determination of how one defines ground truth is an important concept in developing and comparing CAD algorithms. For example, if one circles the tumor nodule and defines everything within that nodule as ground truth "cancer", in actuality the CAD algorithm will be assessed for its ability tumor regions in general, but not the specific tumor cells. For an algorithm such as PPMM, which analyzes lumenal architecture and then scores the general area around these lumens as cancer, circling the tumor would be appropriate. However, if one is evaluating or using a CAD algorithm that assesses specific tumor cytology, circling the tumor area would penalize the algorithm owing to the fact that the circled area would include, in addition to malignant cells of interest: lumenal white spaces, stroma, inflammatory cells, nerves, vascular structures and other adjacent connective tissue (for prostate cancer). These latter categories should all be defined as benign, yet their presence in the ground truth map constitutes a penalty on overall ground truth performance. Therefore, one's ground truth must correspond to the intended specific surface area of interest, as applied to the behavior of the algorithm under consideration.

In addition, when determining ground truth in determining the size of a tumor nodule, one measures the maximal linear dimension. However, prostate cancer is an infiltrative process in which the cancerous glands extend into the surrounding benign glands. Tumor nodules often contain a spectrum of rare to few benign glands. However, if one is making a ground truth map of only the malignant epithelium of prostate cancer, it brings into focus the reality of the tedious and time consuming process required to manually paint all the malignant glands in the tumor nodule. This effort is often compounded by the presence of suspicious looking glands, or atypical glands, which might need confirmatory IHC staining. Therefore, the one making the ground truth map may be compelled to include only those definitively malignant glands based on H\&E cytology (thus forgoing sensitivity) or to include, incrementally, all the suspicious/atypical glands (thus forgoing specificity). Clearly, neither strategy is optimal. Lastly, creating ground truth maps require down-sampling of the WSI data set because current file sizes often exceed the capabilities of most digital image editing tools. We tried "painting" the tumor epithelium of directly onto the digital slide using Aperio's ImageScope tool, but found it was very challenging because its annotation tool produces boundaries, rather than having a "paint brush-like" function as is found in image editing tools that is easily enable the making of pixel-wise truth maps. We found GIMP to be of the greatest utility for editing WSI data sets that were down-sampled $1: 4$ enabling the pathologist to simply "color in" the areas. We envision that PPMM-SIVQ is well positioned to emerge as an effective ground truth generator for malignant prostate epithelium. While no method is perfect, PPMM-SIVQ's current capabilities clearly place the pathologist in the tenable role of merely requiring confirmatory review, with the need for only modest additions/deletions to the initially rendered map, thus saving a significant amount of the pathologist's time. 


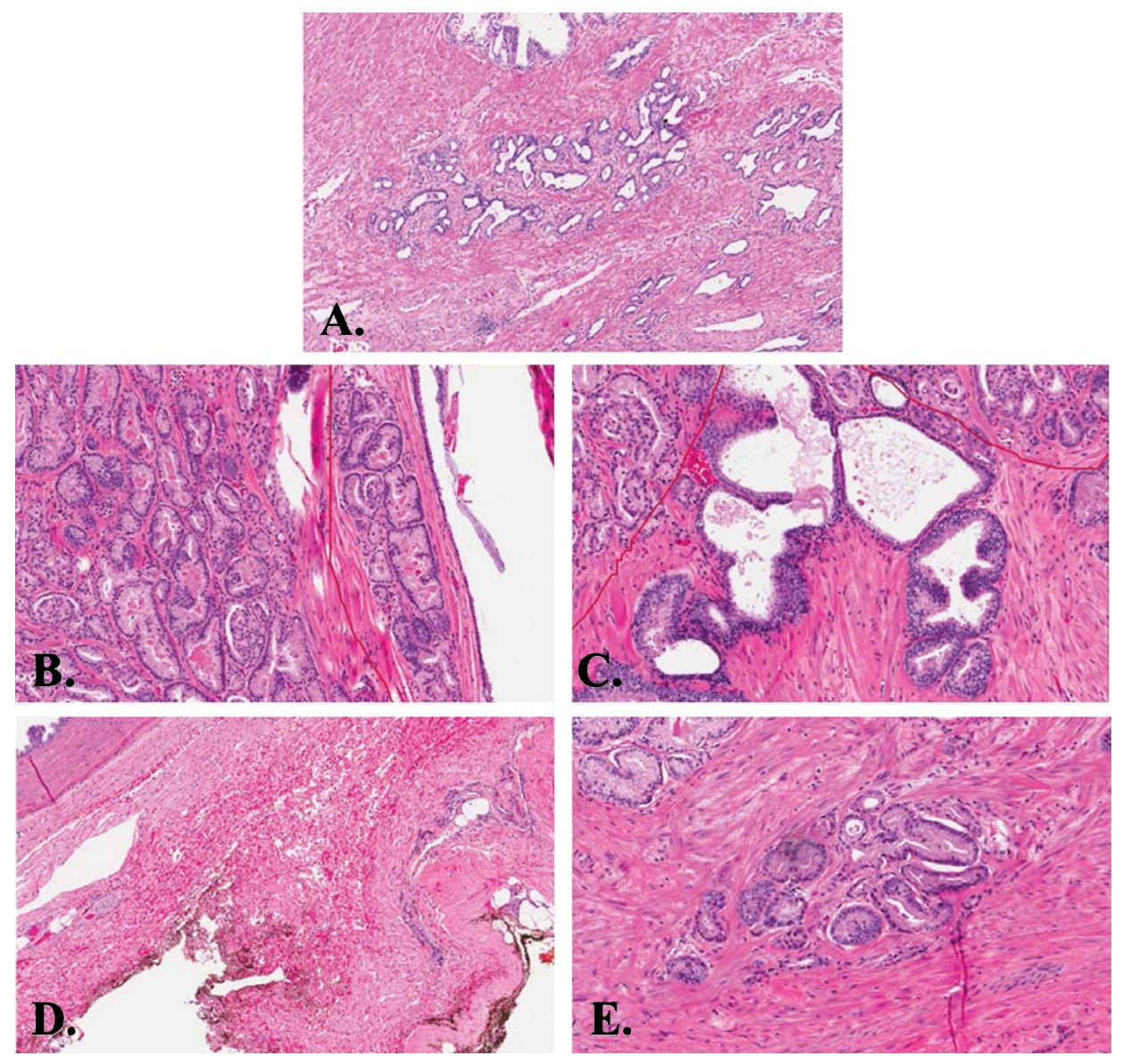

Supplemental Figure 1. Histopathologic features that were or were not identified by PPMM or PPMM-SIVQ. Figure A shows a focus of lobulated small benign atrophic glands from specimen \#1 that was identified as falsely positive by PPMM and PPMM-SIVQ. Figure B shows a focus of medium and large acini that looked very similar to the tumor nodule of specimen \#2 that was identified as positive by PPMM and PPMMSIVQ. Upon further review, it is favored that these glands are malignant and would need immunohistochemistry stains to confirm. Figure C shows prostatic intraepithelial neoplasia (PIN) from specimen \#2 that was falsely positively identified by PPMM and PPMM-SIVQ. Figure D shows two foci of artificial white tissue spaces in specimen \#3 that was falsely identified as positive by PPMM but not PPMM-SIVQ. Figure E shows a minute focus of small and large highly suspicious glands (which was not mapped in the ground truth) and was not identified by PPMM or PPMM-SIVQ. (Colours are visible in the online version of the article; http://dx.doi.org/10.3233/ACP-2012-0054)

\section{Acknowledgments}

This work was made possible via grants from the Wallace H. Coulter Foundation, National Cancer Institute (Grant Nos. R01CA136535-01 and R03CA143991-01), and Burroughs Wellcome Fund (Collaborative Research Travel Grant) supported in part by the Center for Cancer Research of the National Cancer Institute, NIH, Bethesda, MD.

$\mathrm{AM}$ and $\mathrm{JM}$ are majority stockholders in Ibris Inc.

\section{References}

[1] S. Doyle, M.D. Feldman, J.E. Tomaszewski, N. Shih and A. Madabhushi, Cascaded multi-class pairwise classifier (CascaMPa) for normal, cancerous, and cancer confounder classes, In: Prostate histology, IEEE International Symposium on Biomedical Imaging (ISBI) (2011), 715-718.

[2] A.N. Esgiar, R.N. Naguib, B.S. Sharif, M.K. Bennett and Murray A, Microscopic image analysis for quantitative measurement and feature identification of normal and cancerous colonic mucosa, IEEE Trans Inf Technol Biomed 2 (1998), 197-203. 
[3] S. Doyle, A. Madabhushi, M. Feldman and J. Tomaszeweski, A boosting cascade for automated detection of prostate cancer from digitized histology, Med Image Comput Comput Assist Interv 4191 (2006), 504-511.

[4] J. Sudbo, R. Marcelpoil and A. Reith, New algorithms based on the Voronoi Diagram applied in a pilot study on normal mucosa and carcinomas, Anal Cell Pathol 21 (2000), 71-86.

[5] R.M. Haralick, K. Shanmugam and I. Dinstein, Textural features for image classification, IEEE Transactions on Systems, Man, and Cybernetics SMC-3 (1973), 12.

[6] A. Madabhushi, Digital pathology image analysis: Opportunities and challenges, Imaging in Medicine 1(1) (2009), 7-10.

[7] R. Montironi, R. Mazzuccheli, M. Scarpelli, A. Lopez-Beltran, G. Fellegara and F. Algaba, Gleason grading of prostate cancer in needle biopsies or radical prostatectomy specimens: Contemporary approach, current clinical significance and sources of pathology discrepancies, BJU Int 95 (2005), 1146-1152.

[8] W.C. Allsbrook Jr, K.A. Mangold, M.H. Johnson, R.B. Lane, C.G. Lane and J.I. Epstein, Interobserver reproducibility of Gleason grading of prostatic carcinoma: General pathologist, Hum Pathol 32 (2001), 81-88.

[9] C.R. King, Patterns of prostate cancer biopsy grading: Trends and clinical implications, Int J Cancer 90 (2000), 305-311.

[10] M.D. DiFranco, G. O'Hurley, E.W. Kay, R.W. Watson and P. Cunningham, Ensemble based system for whole-slide prostate cancer probability mapping using color texture features, Comput Med Imaging Graph 35 (2011), 629-645.

[11] S. Doyle, M. Feldman, J. Tomaszewski and A. Madabhushi, A boosted bayesian multi-resolution classifier for prostate cancer detection from digitized needle biopsies, IEEE Trans Biomed Eng June 2199 (2010).

[12] J.P. Monaco, J.E. Tomaszewski, M.D. Feldman, I. Hagemann, M. Moradi, P. Mousavi, A. Boag, C. Davidson, P. Abolmaesumi and A. Madabhushi, High-throughput detection of prostate cancer in histological sections using probabilistic pairwise Markov models, Med Image Anal 14 (2010), 617-629.

[13] J.D. Hipp, J.Y. Cheng, M. Toner, R. Tompkins and U. Balis, Spatially invariant vector quantization: A pattern matching algorithm for multiple classes of image subject matter- including pathology, J Pathol Inform 2 (2010), 13.

[14] J. Hipp, J. Cheng, S. Daignault, J. Sica, M.C. Dugan, D. Lucas, Y. Yagi, S. Hewitt and U.J. Balis, Automated area calculation of histopathologic features using SIVQ analytical cellular pathology, Pending Publication.

[15] J. Hipp, J. Cheng, J.C. Hanson, W. Yan, P. Taylor, N. Hu, J. Rodriguez-Canales, J. Hipp, M.A. Tangrea, M.R. EmmertBuck and U. Balis, SIVQ-aided laser capture microdissection: A tool for high-throughput expression profiling, Journal of Pathology Informatics 2 (2011), 19.

[16] C. Chargari, E. Comperat, N. Magne, L. Vedrine, A. Houlgatte, L. Egevad and P. Camparo, Prostate needle biopsy examination by means of virtual microscopy, Pathol Res Pract 207 (2011), 366-369.

[17] P.W. Huang and C.H. Lee, Automatic classification for pathological prostate images based on fractal analysis, IEEE Trans Med Imaging 28 (2009), 1037-1050.
[18] A. Tabesh, M. Teverovskiy, H.Y. Pang, V.P. Kumar, D. Verbel, A. Kotsianti and O. Saidi, Multifeature prostate cancer diagnosis and Gleason grading of histological images, IEEE Trans Med Imaging 26 (2007), 1366-1378.

[19] P.W. Hamilton, P.H. Bartels, R. Montironi, N.H. Anderson, D. Thompson, J. Diamond, S. Trewin and H. Bharucha, Automated histometry in quantitative prostate pathology, Anal Quant Cytol Histol 20 (1998), 443-460.

[20] J.D. Hipp, D.R. Lucas, M.R. Emmert-Buck, C.C. Compton and U.J. Balis, Digital slide repositories for publications: Lessons learned from the microarray community, Am J Surg Pathol 35 (2011), 783-786.

[21] J.D. Hipp, J. Sica, B. McKenna, J. Monaco, A. Madabhushi, J. Cheng and U.J. Balis, The need for the pathology community to sponsor a whole slide imaging repository with technical guidance from the pathology informatics community, J Pathol Inform 2 (2011), 31.

[22] B.A. Nelson, S.B. Shappell, S.S. Chang, N. Wells, S.B. Farnham, J.A. Smith and M.S. Cookson, Tumour volume is an independent predictor of prostate-specific antigen recurrence in patients undergoing radical prostatectomy for clinically localized prostate cancer, BJU Int 97 (2006), 1169-1172.

[23] L.E. Eichelberger, M.O. Koch, J.N. Eble, T.M. Ulbright, B.E. Juliar and L. Cheng, Maximum tumor diameter is an independent predictor of prostate-specific antigen recurrence in prostate cancer, Mod Pathol 18 (2005), 886-890.

[24] G.F. Carvalhal, P.A. Humphrey, P. Thorson, Y. Yan, C.G. Ramos and W.J. Catalona, Visual estimate of the percentage of carcinoma is an independent predictor of prostate carcinoma recurrence after radical prostatectomy, Cancer 89 (2000), 1308-1314.

[25] A.A. Renshaw, J.P. Richie, K.R. Loughlin, M. Jiroutek, A. Chung and A.V. D'Amico, Maximum diameter of prostatic carcinoma is a simple, inexpensive, and independent predictor of prostate-specific antigen failure in radical prostatectomy specimens. Validation in a cohort of 434 patients, Am J Clin Pathol 111 (1999), 641-644.

[26] B.I. Chung, T.V. Tarin, M. Ferrari and J.D. Brooks, Comparison of prostate cancer tumor volume and percent cancer in prediction of biochemical recurrence and cancer specific survival, Urol Oncol (2009).

[27] P.A. Humphrey and R.T. Vollmer, Percentage carcinoma as a measure of prostatic tumor size in radical prostatectomy tissues, Mod Pathol 10 (1997), 326-333.

[28] R.T. Vollmer, Percentage of tumor in prostatectomy specimens: A study of American Veterans, Am J Clin Pathol 131 (2009), 86-91.

[29] G. Xiao, B.N. Bloch, J. Chappelow, E.M. Genega, N.M. Rofsky, R.E. Lenkinski, J. Tomaszewski, M.D. Feldman, M. Rosen and A. Madabhushi, Determining histology-MRI slice correspondences for defining MRI-based disease signatures of prostate cancer, Comput Med Imaging Graph 35(7-8) (2011), $568-578$. 


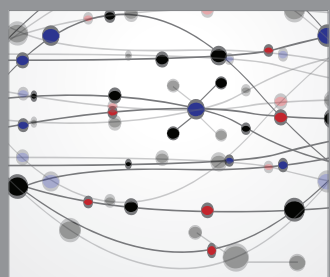

The Scientific World Journal
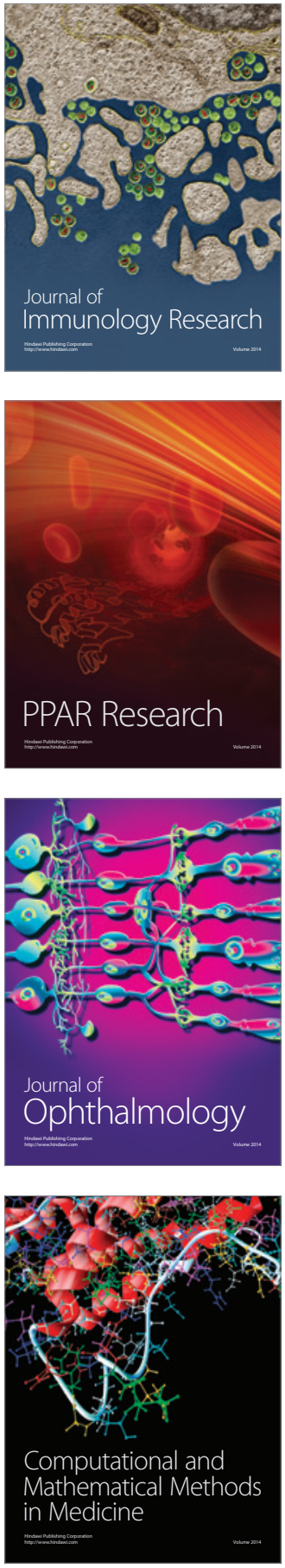

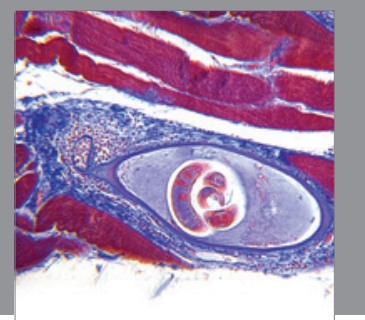

Gastroenterology

Research and Practice
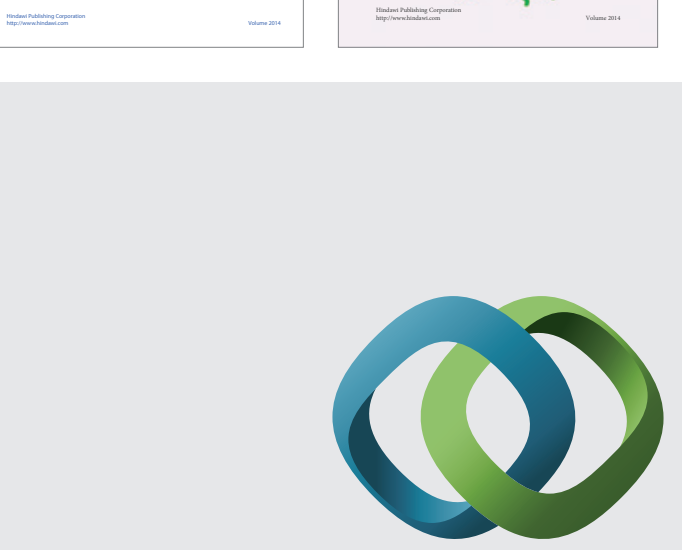

\section{Hindawi}

Submit your manuscripts at

http://www.hindawi.com
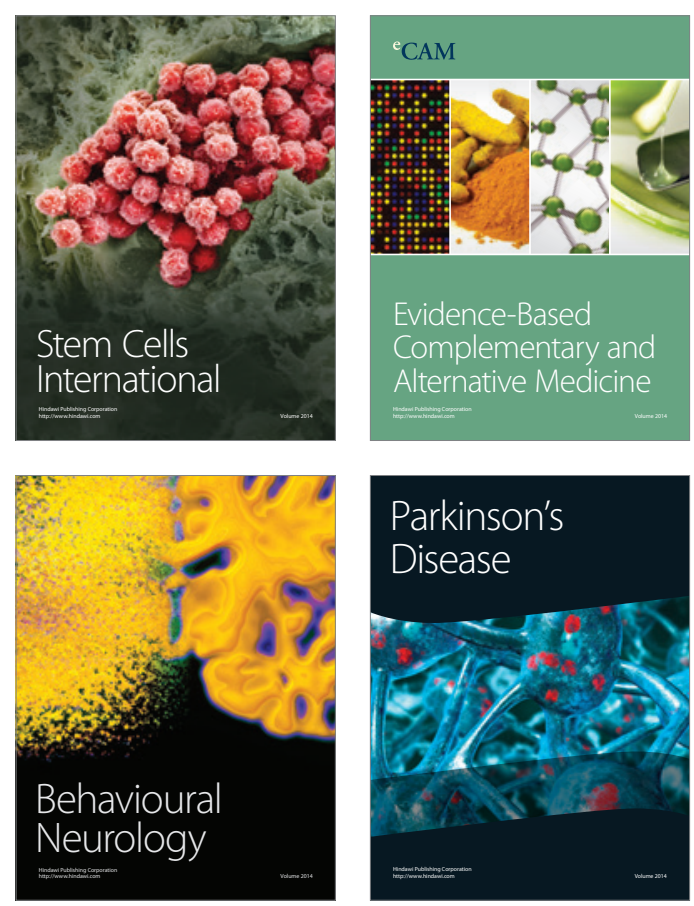

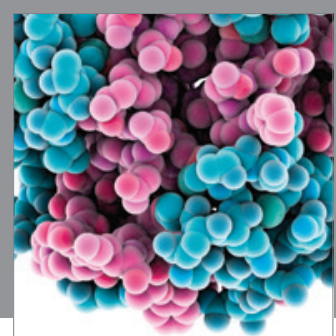

Journal of
Diabetes Research

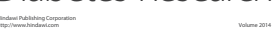

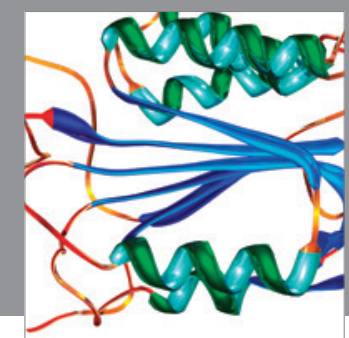

Disease Markers
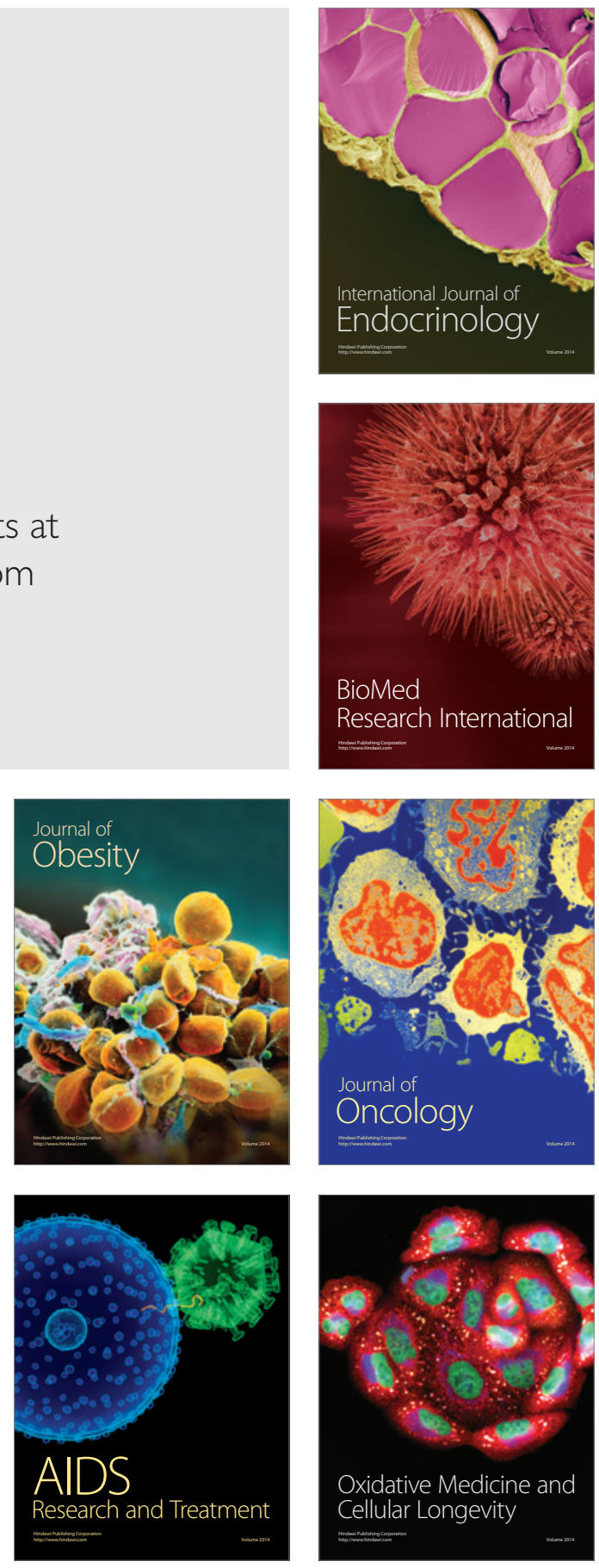